\title{
Effect of trans-10, cis-12 isomer of conjugated linoleic acid on boar semen quality after cryopreservation
}

\author{
Sofia Margarida Pontes Teixeira ${ }^{1}$, António Eduardo Nobre Chaveiro, Joaquim Fernando Moreira da Silva
}

University of the Azores, Department of Agrarian Sciences, Animal Reproduction, CITA-A, Angra do Heroísmo, Portugal.

\begin{abstract}
The use of frozen semen in pig industry is limited by problems with viability and fertility compared to cooled semen. Part of the decrease in motility and fertility, associated to cryopreservation, may be due to oxidative damage from excessive formation of reactive oxygen species (ROS). Frozen thawed boar spermatozoa are still considered suboptimal due to the low conception rates and smaller litters after artificial insemination. The relatively low fertility of frozen thawed boar semen is associated with many factors including cytotoxicity of the cryoprotectant, osmotic stress, injuries due to ice formation during freezing and thawing, cold shock damages and even inter and intra variations present among boars. Therefore, this study was conducted to determine the impact of conjugated linoleic acid (trans10, cis-12; CLA) supplementation in the cryopreservation extender frozen-thawed boar on semen quality parameters. Semen was collected from three boars (three ejaculates per boar) which were subjected to cryopreservation, without any supplementation (control) or supplemented with $50 \mu \mathrm{m}$ CLA, and then the semen was frozen using a controlled rate freezer. Before freezing, and after thawing, the sperm motility was assessed, microscopically and viability and acrosome integrity were assessed using the flow cytometry technique. Regarding live spermatozoa, no significant differences $(\mathrm{P}>0.05)$ were observed among treatments. However, statistical differences $(\mathrm{P}<0.05)$ were found between refrigerated and frozen-thawed semen. Both sperm viability and motility diminished after thawing. Significant differences $(P<0.05)$ in motility were found not only between refrigerated semen and frozen-thawed group, but also between treatments. In acrosome integrity, no significant differences $(P>0.05)$ were observed among treatments. In conclusion, the addition of trans-10, cis-12 isomer of conjugated linoleic acid, in the concentration used in the cryopreservation media, showed no advantages on the post-thaw boar sperm viability and integrity.
\end{abstract}

Keywords: boar, conjugated linoleic acid, cryopreservation, flow cytometry, semen.

\section{Introduction}

The use of artificial insemination (AI) is commonly used in the modern swine breeding industry. However, AI utilizing cryopreserved semen accounts for less than $1 \%$ of total inseminations performed due to low conception rates and reduced litter sizes (Zeng et al., 2014). This could be due to the process of cooling, freezing, and thawing, causing physical and chemical stress on sperm membranes, which diminishes sperm viability and fertilizing ability (Gadea et al., 2005).

In order to improve cryopreservation technology, several studies have focused on understanding the mechanisms behind cryodamages, which have been shown that the extent of the cryodamage on semen is dependent on many factors, such as extender composition, freezing protocols and individual variability (Fraser et al., 2014). Although significant advances have been made in improving boar spermatozoa cryopreservation, the freezing extenders that are currently being used are still considered to be suboptimal, and the mechanisms contributing to the cryoinjury of boar spermatozoa remain to be clarified. Cryopreserved mammalian semen is generally acknowledged to have reduced fertility compared to fresh semen (Watson, 2000).

Boar spermatozoa are especially sensitive to cold temperatures due to the high content of polyunsaturated fatty acids (PUFAs) and the low antioxidant capacity of seminal plasma (Cerolini et al., 2000). The membrane lipid bilayer of boar semen presents differences in relation to other species that could explain the increased susceptibility to cold shock. Among the major differences are a lower percentage of cholesterol molecules and their asymmetrical distribution in the membrane with a higher amount in the inner than the external monolayer. These structural differences could aid in understanding this reaction during cold shock (Shiomi, 2013). Cryopreservation techniques have been greatly improved in recent years allowing for good results in terms of thawed sperm quality. However, that quality varies widely between boars and even between ejaculates.

Some fatty acid has been supplemented into the semen extenders, in different species, to try to minimize ROS formation and also to protect the plasma membrane function (Kaeoket, 2012). Antioxidants exert a protective effect on the plasma membrane of frozen boar sperm and among different antioxidants, conjugated linoleic acid (CLA) is the terminology used to define a group of isomers of octadecadienoic acid with double conjugated bonds, that are most plentiful in positions $9,10,11$, and 12 CLA, as essential fatty acids (linoleic and linolenic acids), and other PUFAs, are known for changing the lipid membrane composition in many cells (Sampath and Ntambi, 2005). These fatty acids can be incorporated into the plasma membrane of 
the cells, causing modifications in structure and function (Ringseis et al., 2008; Amaru and Field, 2009), and can influence the membrane stability during cryopreservation. Whereas relatively few studies have reported the effect of unsaturated fatty acids on sperm membranes, in this study we evaluated the effect of the addition of trans-10, cis-12 isomer of conjugated linoleic acid (CLA) to the cryopreservation extender on the membrane integrity and viability of boar semen submitted to cryopreservation process.

\section{Material and Methods}

The use and care of boars in this study was approved by the Author's Institution's Ethics Committee and the experimental procedures described herein were approved by this committee.

Unless otherwise noted, all reagents were purchased from Sigma Chemical (St. Louis, MO, USA).

\section{Semen collection and processing}

Semen was collected from three boars (three ejaculates per boar) using the gloved-hand method. Then, the semen was diluted in Beltsville Thawing Solution (BTS; Gadea, 2003) extender (preheated at $37^{\circ} \mathrm{C}$ ) and transported to the laboratory for evaluation and processing. Only ejaculates containing more than $75 \%$ motile sperm (evaluated by contrast-phase microscopy) were submitted to the cryopreservation process

\section{Freezing and thawing process}

Semen samples were processed using the straw-freezing procedure described by Westendorf et al. (1975) with minor modifications. Diluted semen was placed at $15^{\circ} \mathrm{C}$ for $2 \mathrm{~h}$ and centrifuged at $800 \mathrm{x}$ g for 10 min. The supernatant was discarded, and sperm counts of the pellets were performed in a Neubauer chamber. The semen pellet was resuspended with lactose egg yolk (LEY) extender $(80 \mathrm{ml}$ of $11 \%$ lactose and $20 \mathrm{ml}$ egg yolk) to provide $1.5 \times 10^{9}$ spermatozoa $/ \mathrm{ml}$ and divided into two aliquots corresponding to different treatments: without any supplementation (control); and supplemented with CLA [Conjugated Linoleic Acid (10 trans, 12 cisCLA; Matreya, catalog: 1249-1, 10(E), 12(Z)Octadecadienoic acid)] in final concentration of $50 \mu \mathrm{M}$. After further cooling to $5^{\circ} \mathrm{C}$ for $120 \mathrm{~min}$, two parts of LEY extender semen were mixed with LEY extender with $1.5 \%$ Orvus Es Paste (Equex-Paste, Minitub, Tiefenbach, Germany) and $9 \%$ glycerol. The final concentration of semen to be frozen was $1 \times 10^{9}$ spermatozoa $/ \mathrm{ml}$ and $3 \%$ glycerol. Afterwards, the diluted and cooled semen was packed in $0.5 \mathrm{ml}$ French straws and frozen in a controlled rate freezer (IceCube 14S; SyLab, Austria). The freezing rate was $1^{\circ} \mathrm{C} / \mathrm{min}$ from $5^{\circ} \mathrm{C}$ to $-4.5^{\circ} \mathrm{C}, 1 \mathrm{~min}$ at $-4.5^{\circ} \mathrm{C}$, and then $30^{\circ} \mathrm{C} / \mathrm{min}$ from $-4.5^{\circ} \mathrm{C}$ to $-100^{\circ} \mathrm{C}$. The straws were then stored in liquid nitrogen until thawing. Thawing was carried out by immersing the straws in a circulating water bath at $52^{\circ} \mathrm{C}$ for $12 \mathrm{sec}$. Immediately after thawing, the semen was diluted in the thawing media (BTS) at $37^{\circ} \mathrm{C}$ and kept in these media for $30 \mathrm{~min}$ before being assayed (Gadea et al., 2005)

\section{Sperm evaluation}

Before freezing and after thawing, different parameters of the cell's sperm activity, such as viability and acrosomal status, were measured using a FACSCalibur flow cytometer (Becton Dickinson, San Jose, CA) after excitation at $469 \mathrm{~nm}$ and emission of green fluorescence at $541 \mathrm{~nm}$ through a $15-\mathrm{mW}$ argon ion laser, at a rate of 500-1000 cells/s, using FACSflow as sheath fluid. Data were then analyzed using Cellquest software (Becton Dickinson). Mean fluorescence intensity of the analyzed sperm cells $(n=10,000)$ was determined after getting the cell population by forward and side light scatter signals.

\section{Evaluation of viability}

Combination of the fluorochromes Sybr-14 and propidium iodide (PI; Live-Dead-Sperm Viability Kit L7011; Invitrogen, Eugene, OR, USA) was used to determine the viability of the sperm, as in Teixeira et al. (2015a).

Briefly, a staining solution was prepared by adding $5 \mu \mathrm{l}$ of a $2 \mathrm{mM}$ solution of PI (in water) and $2 \mu \mathrm{l}$ of a $100 \mathrm{mM}$ solution of SYBR-14 (in anhydrous DMSO) to $2 \mathrm{ml}$ of BTS. Just prior to measurement, 495 $\mu \mathrm{l}$ of staining solution was added to $5 \mu \mathrm{l}$ of semen and incubated for $15 \mathrm{~min}$, at room temperature, in the dark, and then analyzed by flow cytometry.

\section{Evaluation of acrosome integrity}

Evaluation of acrosome integrity was performed by staining sperm with PI and pisum sativum agglutinin conjugated to fluorescein isothiocinate (FITCPSA). The semen was diluted in PBS to $1 \times 10^{6} \mathrm{sperm} / \mathrm{ml}$ in a final volume of $2 \mathrm{ml}$. Then, $296 \mu \mathrm{l}$ of this dilution was withdrawn and placed in a cytometric tube, preheated to $37^{\circ} \mathrm{C}$, stained with $6 \mu \mathrm{l}$ of PI $(0.5 \mathrm{~g} / \mathrm{ml})$ and $20 \mu \mathrm{l}$ of FITC-PSA $(100 \mathrm{mg} / \mathrm{ml})$, and then incubated for $10 \mathrm{~min}$ at $37^{\circ} \mathrm{C}$. Immediately before analyzing, $300 \mu \mathrm{l}$ of BTS was added and samples were measured by flow cytometry, as described by Teixeira et al. (2015b). Sperm was then allocated to one of four groups based on FITCPSA and PI staining patterns: i) FICT-PSA negative and PI negative, acrosome intact live (AIL); ii) FICT-PSA negative and PI positive, acrosome intact dead (AID); iii) FICT-PSA positive and PI negative, acrosome reacted/damaged live (ARL); and iv) FICT-PSA positive and PI positive, acrosome damaged dead (ARD).

\section{Statistical analysis}

Comparisons of semen parameters between different groups and treatments were analyzed by oneway analysis of variance (ANOVA) and expressed as means \pm SEM. Percentile data were normalized through arc sine transformation and the data was then submitted to a homogeneity test, followed by variance analysis (one-way ANOVA), with posthoc Least significant difference test by IBM SPSS version 20 Statistics Program software (SPSS Inc. Chicago, IL). 
For all analyses, a $\mathrm{P}$ value of $\leq 0.05$ was considered statistically different.

\section{Results}

In the present study, the effect of the addition of CLA in the dilution and cryopreservation extender was analyzed.

The percentages of motile sperm (Fig. 1) were higher in refrigerated semen compared to the frozen-thawed semen, presenting significant differences $(\mathrm{P}<0.05)$. Also, when the two groups are compared, the control has higher rates than the CLA supplementation group.

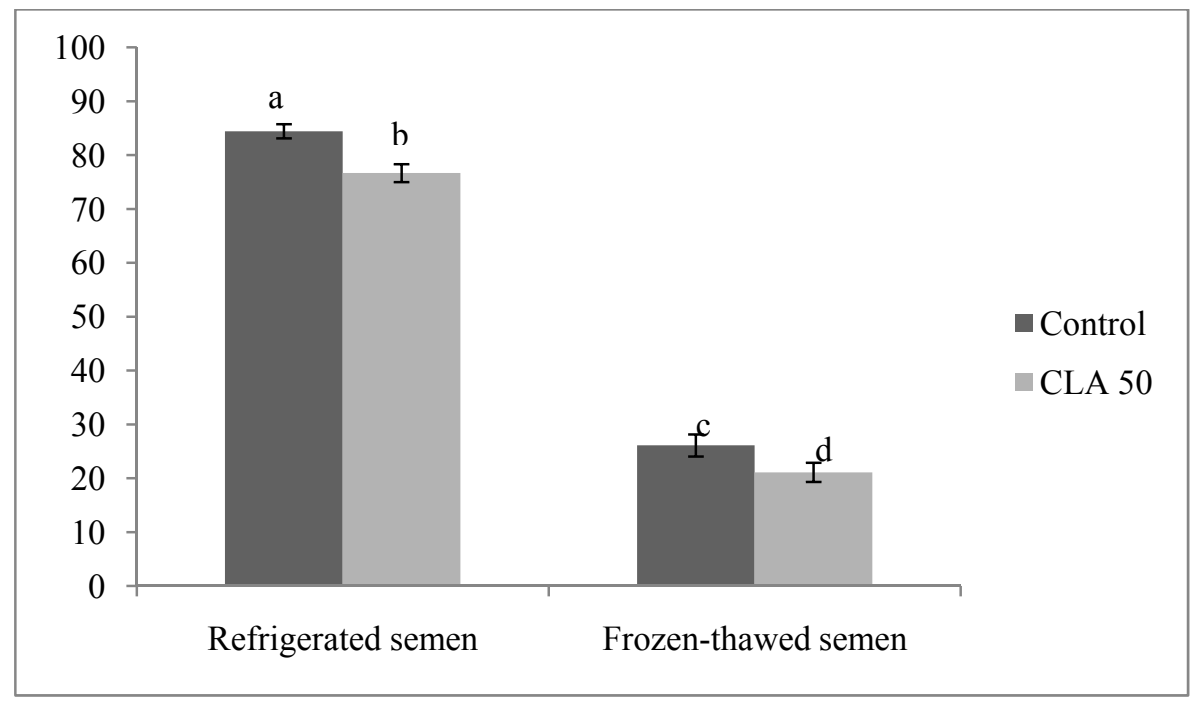

Figure 1. Percentage of sperm motility (mean $\pm \mathrm{SE}$ ) of refrigerated and frozen-thawed boar semen. ${ }^{\mathrm{a}, \mathrm{b}, \mathrm{c}, \mathrm{d}}$ Different superscripts indicates significant differences, $\mathrm{P} \leq 0.05$.

A significant loss of sperm viability was observed when the semen was frozen-thawed (Fig. 2). Furthermore, no statistically significant differences $(\mathrm{P}>$ $0.05)$ were observed between the control and the CLA supplemented extender, either in refrigerated semen or frozen-thawed semen.

As far as the integrity of acrosome is concerned, spermatozoa were allocated to one of four groups on the basis of their FITC-PSA and PI staining patterns (Fig. 3), as follows: 1) FICT-PSA negative and PI negative, acrosome intact live sperm (AIL); 2) FICT-
PSA positive and PI negative, acrosome reacted live sperm (ARL); 3) FICT-PSA negative and PI positive, acrosome intact dead (AID); and 4) FICT-PSA positive and PI positive, acrosome intact dead sperm (ARD). For all sperm populations of boar sperm, no significant differences were observed between the control group and the CLA supplementation in both refrigerated and frozen-thawed semen. Despite the lack of differences between the treatments, there were statistically significant differences $(\mathrm{P}<0.05)$ when the refrigerated and frozen-thawed semen were compared.

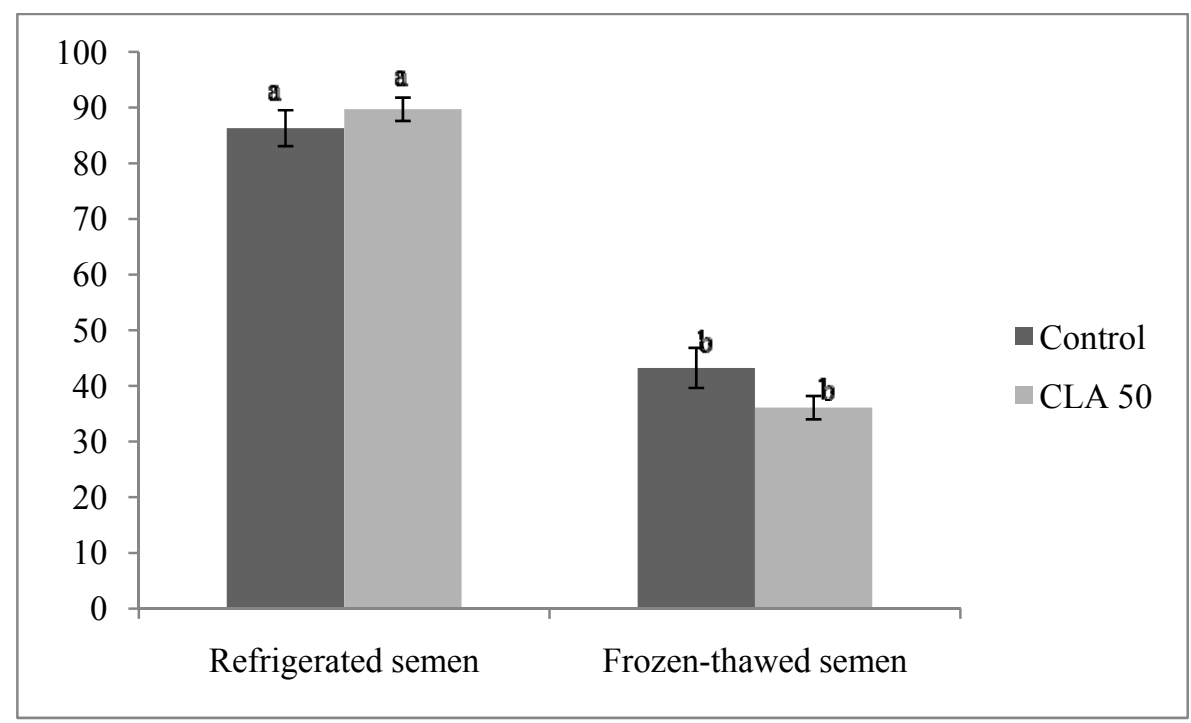

Figure 2. Refrigerated and frozen-thawed boar sperm viability (mean $\pm \mathrm{SE}$ ), with and without CLA (50 $\mu \mathrm{m})$. ${ }^{\mathrm{a}, \mathrm{b}}$ Different superscript within the same row indicates significant differences, $\mathrm{P} \leq 0.05$. 


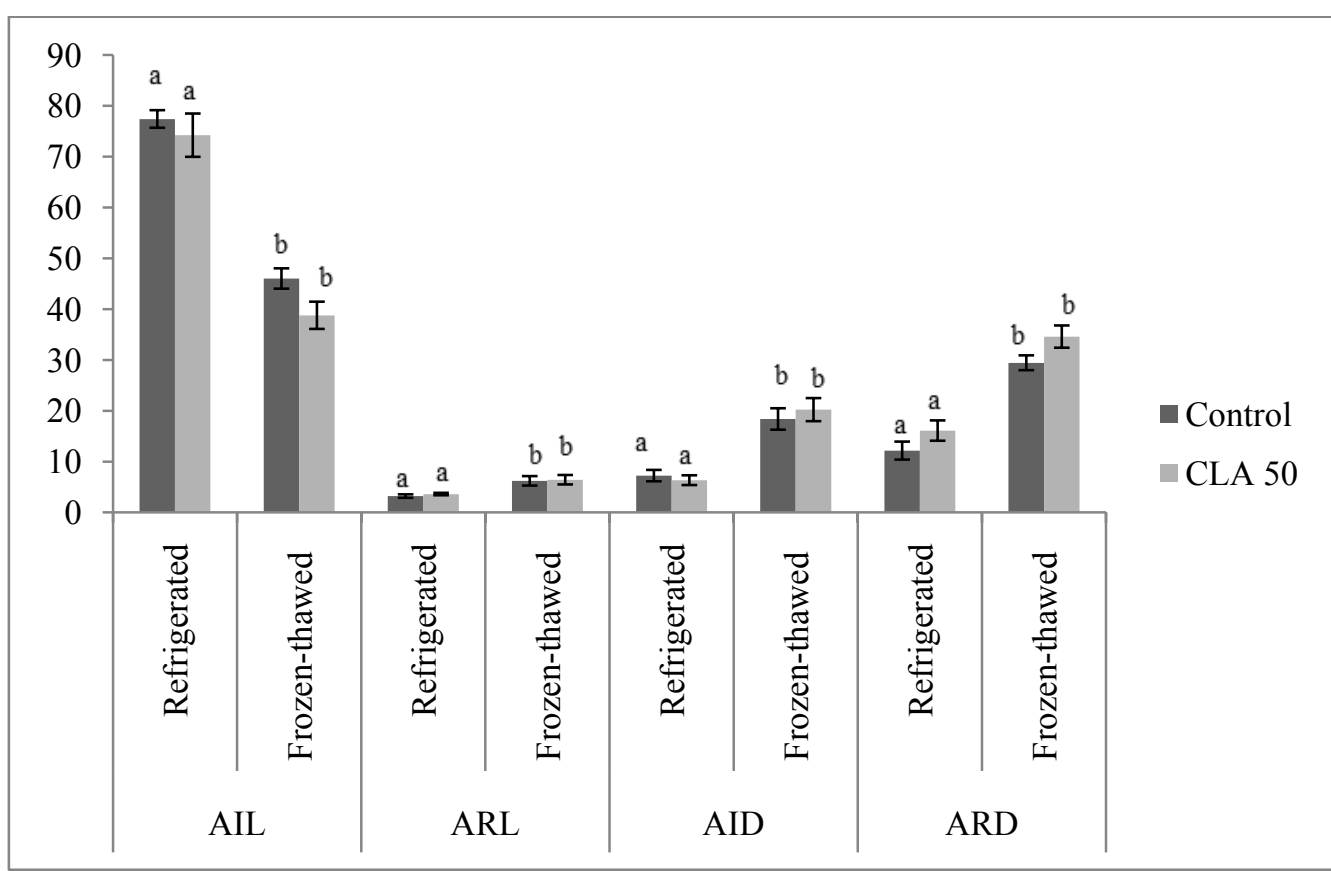

Figure 3. Evaluation by flow cytometry of refrigerated and thawed boar sperm acrosome status (mean $\pm \mathrm{SE}$ ); acrosome intact and live cells (AIL), acrosome reacted and live cells (ARL), acrosome intact and dead cells (AID), and acrosome degenerate and dead cells (ARD) with and without CLA $(50 \mu \mathrm{m})$. ${ }^{\mathrm{a}, \mathrm{b}}$ Different superscript within the same row indicates significant differences, $\mathrm{P} \leq 0.05$.

\section{Discussion}

In an attempt to minimize the detrimental effects of the oxidative stress, the impact of CLA supplementation in the refrigeration and cryopreservation extender of refrigerated and frozenthawed boar semen was investigated by studying parameters such as motility, viability and acrosome integrity of refrigerated and frozen-thawed boar sperm in the presence of CLA. Significant differences among treatments were detected in terms of sperm motility (Fig. 1), in refrigerated sperm and also after thawing, suggesting that the presence of CLA did not improve the motility of cryopreserved boar sperm. Although the effects of fatty acids during the freezing of boar spermatozoa have not been previously investigated, Hossain et al. (2007), observed an increase in boar sperm motility after the addition of oleic, linoleic and arachidonic acids into the dilution medium. The effects of CLA addition to dilution and freezing media used for boar semen and its interaction with sperm cells have not been reported, so the addition of fatty acids in semen cryopreservation media may influence sperm motility after thawing, possibly by maintaining membrane fluidity due to their incorporation in lipid bilayers.

In terms of viability (Fig. 2), the results show that, despite having high percentages of viable refrigerated semen, they decreased significantly after freezing and thawing, indicating that one of these two steps, or both, can be lethal for spermatozoa.

According to Ohata et al. (2001) the equilibrium period, characterized by the slow reduction of temperature after collection and before submitting samples to temperatures below $15^{\circ} \mathrm{C}$, aims to reduce the harmful effects of heat shock on sperm, which increases its viability after thawing. As it would be expected, the motility and viability of refrigerated spermatozoa were significantly higher than those of frozen-thawed sperm. This decrease in functional abilities could be due to cold shock (White, 1993), intracellular ice formation, and/or osmotic stress (Watson, 1995), which causes damage to the sperm membrane, mitochondria, acrosome, and sperm tail. Cryopreservation, and also refrigeration, are known to decrease motility (Maldjian et al., 2005) causing structural damage to plasma and acrosomal membranes and decreasing functional integrity and sperm survival rates (Salamon and Maxwell, 2000). Cryopreservation has also been shown to lower mitochondrial function, potentially interrupting ATP availability to the tail filaments indicating the mitochondria as perhaps the most sensitive of the sperm structure (Teixeira et al. 2015a).

Acrosome reactions are related to sperm fertility and are necessary for the fertilizing capacity of spermatozoa (Birck et al., 2009). Analyzing sperm acrosome integrity through cytometry may be useful in predicting the potential fertility of boars. Our results related to acrosome integrity (Fig. 3) showed that a significantly higher percentage of spermatozoa with intact acrosomes were found in refrigerated semen compared to the frozen-thawed group. This supports the findings of Barros et al. (2012), who also achieved better results with refrigerated semen. Ohata et al. (2005) reported higher percentages of acrosome integrity when they incubated semen for more $h$, showing that pre-freezing for $20 \mathrm{~h}$ provides acrosome with greater stability after thawing and reduces the harmful effects of thermal shock on sperm.

According to Perez et al. (1996), spermatic cells that survive the freezing/thawing process present 
cryopreservation induced alterations, resulting in a sperm population with decreased lifetime and increased mitochondrial damage, which is likely the main reason for the lower quality of thawed semen.

It has been reported that mitochondrial activity is related to sperm motility. Energy is essential for sperm motility and fertilization is delivered in the form of ATP partly synthesized through oxidative phosphorylation in the mitochondria (Mahadevan et al., 1997). Reduced sperm motility may be related to the fact that the plasma membrane of boar spermatozoa is extremely sensitive to changing temperatures during the cooling, freezing and thawing process (Watson, 2000), which might lead to the weakening of mitochondrial function. In contrast, Marin et al. (2003) stated that boar spermatozoa used less energy from mitochondria for motility than dog spermatozoa. The presence of high levels of PUFAs in the plasma membrane of boar spermatozoa creates favorable conditions for the formation of peroxidative products, major damage that may ultimately impair fertility. Part of the reduction in sperm motility and fertility associated with cryopreservation may be due to the oxidative damage from excessive ROS (Guthrie and Welch 2006).

Fatty acids, can influence the stability of the membranes facing refrigeration and freezing. Action mechanism and molecular basis for the different effects of CLA isomers remains unclear despite numerous experimental studies by Zhao et al. (2011). It is possible that at least some of the CLA effects occur due to changes in membrane structure and function (Takahashi et al., 2012), after incubating bull sperm with low freezability with linoleic acid for $30 \mathrm{~h}$ and then freeze and thawed, observed high motility and described that the beneficial effects of using fatty acids in semen dilution should be the possibility of maintaining the fluidity of membrane and are assigned to the incorporation of linoleic acid to membrane. Also Pérez-Pé et al. (2001), added oleic-linoleic acids to the cryopreservation medium of ovine semen which resulted in a beneficial effect in the preservation of sperm cells viability. In our previously study (Teixeira et al. 2015a) it has been found that the viability was improved in concentrations of $50 \mu \mathrm{M}$ CLA, so the same concentration was used in the current experiment to cryopreserve the semen, which had no clear positive outcomes on post-thaw boar sperm integrity and viability.

In conclusion, results obtained in this study, clearly demonstrated that addition of trans-10, cis- 12 isomer of conjugated linoleic acid (CLA) to the freezing extender does not improve the viability of boar spermatozoa after thawing. These results suggest that the role of oxidative stress in cryopreservation inducing damage of boar spermatozoa requires further investigation.

\section{Acknowledgments}

The first author is supported by the Regional Fund of Science Grant BD M3.1.2/F/034/2011. Serviços de Desenvolvimento Agrário da Ilha Terceira, and particularly Mr. Jorge Armas, are fully acknowledged for their help during semen collection. CITA-A is also fully acknowledged.

\section{References}

Amaru DL, Field CJ. 2009. Conjugated linoleic acid decreases mcf-7 human breast cancer cell growth and insulin-like growth factor-1 receptor levels. Lipids, 44:449-458.

Barros MHC, Shiomi HH, Amorim LS, Guimarães SEF, Lopes PS, Siqueira JB, Guimarães JD. 2012. Criopreservação de sêmen de suínos da raça Piau submetido a três protocolos de congelamento. Rev Bras Zootec, 41:914-922.

Birck A, Labouriau R, Christensen P. 2009. Dynamics of the induced acrosome reaction in boar sperm evaluated by flow cytometry. Anim Reprod Sci, 115:124-136.

Cerolini S, Maldjian A, Surai P, Noble R. 2000. Viability susceptibility to peroxidation and fatty acid composition of boar semen during liquid storage. Anim Reprod Sci, 1-2:99-111.

Fraser L, Strzezek J, Kordan W. 2014. Post-thaw sperm characteristics following long-term storage of boar semen in liquid nitrogen. Anim Reprod Sci, 147:119-127.

Gadea J. 2003. Semen extenders used in artificial insemination of swine. Span J Agric Res, 1:17-27.

Gadea J, Gumbao D, Matas C, Romar R. 2005. Supplementation of the thawing media with reduced glutathione improves function and the in vitro fertilizing ability of boar spermatozoa after cryopreservation. $J$ Androl, 26:749-756.

Guthrie HD, Welch GR. 2006. Determination of intracellular reactive oxygen species and high mitochondrial membrane potential in Percoll-treated viable boar sperm using fluorescence-activated flow cytometry. J Anim Sci, 84:2089-2100.

Hossain MDS, Tareq KMA, Hammano K, Tsuji H. 2007. Effect of fatty acids on boar sperm motility, viability and acrossome reaction. Reprod Med Biol, 6:235-239.

Kaeoket K. 2012. Cryopreservation of boar spermatozoa: an important role of antioxidants. In: Katkov I (Ed.). Current Frontiers in Cryopreservation. Rijeka, Croatia: InTech. pp. 139-164.

Mahadevan MM, Miller MM, Moutos DM. 1997. Absence of glucose decreases human fertilization and sperm movement characteristic in vitro. Hum Reprod, 12:119-123.

Maldjian A, Pizzi F, Gliozzi T, Cerolini S, Penny P, Noble R. 2005. Changes in sperm quality and lipid composition during cryopreservation of boar semen. Theriogenology, 63:411-421.

Marin S, Chiang K, Bassilian S, Lee WN, Boros LG, Fernandez-Novell JM, Centelless JJ, Medrano A, Rodriguez-Gil JE, Cascante M. 2003. Metabolic strategy of boar spermatozoa revealed by metabolomic characterization. FEBS Lett, 554:342-346.

Ohata PM, Wentz I, Bernardi ML. 2001. Viability of frozen swine semen submitted to a pre-freezing equilibrium time in the presence or absence of seminal 
plasma. Arq Fac Vet UFRGS, 29:123-129.

Ohata PM, Bernardi ML, Reis GR. 2005. Congelabilidade do sêmen suíno de acordo com o período de equilíbrio pré-congelamento e da sensibilidade ao resfriamento. Arch Vet Sci, 10:69-74.

Pérez LJ, Valcárcel A, de las Heras MA, Moses D, Baldassarre H. 1996. Evidence that frozen/thawed ram spermatozoa show accelerated capacitation in vitro as assessed by chlortetracycline assay. Theriogenology, 46:131-140.

Pérez-Pé R, Cebrian-Pérez JA, Muiño-Blanco $\mathbf{T}$. 2001.Semen plasma proteins prevent cold-shock membrane damage to ram spermatozoa, Theriogenology, 56:425-434.

Ringseis R, Gaiping W, Saal D, Eder K. 2008. Conjugated linoleic acid isomers reduce cholesterol accumulation in acetylated LDL-induced mouse RAW264.7 macrophage derived foam cells. Lipids, 43:913-923.

Salamon S, Maxwell WMC. 2000. Storage of ram semen. Anim Reprod Sci, 62:77-111.

Sampath H, Ntambi JM. 2005. Polyunsaturated fatty acid regulation of genes of lipid metabolism. Annu Rev Nutr, 25:317-340.

Shiomi HH. 2013. Criopreservação de espermatozoides suínos da raça Piau: avaliação de curvas de congelamento e centrifugações. Viçosa, MG: Universidade Federal de Viçosa. Thesis.

Takahashi T, Itoh R, Nishinomiya H, Katoh M, Manabe N. 2012. Effect of linoleic acid albumin in a dilution solution and long-term equilibration for freezing of bovine spermatozoa with poor freezability. Reprod Domest Anim, 47:92-97.
Teixeira SMP, Chaveiro A, Silva FM. 2015a. Effect of conjugated linoleic acid on boar semen quality after long-term refrigeration at $17^{\circ} \mathrm{C}$. Reprod Domest Anim, 50:604-610.

Teixeira SMP, Chaveiro A, Silva FM. 2015b. The effects of three extenders on refrigerated boar semen. Afr J Anim Sci, 45:82-88.

Trout SW. 2012. Evaluation of different concentrations of egg yolk in canine frozen semen extender. Blacksburg, VA: Faculty of Virginia Polytechnic Institute and State University. Master of Science Thesis. Watson PF. 1995. Recent developments and concepts in the cryopreservation of spermatozoa and the assessment of their post-thawing functions. Reprod Fertil Dev, 7:871-891.

Watson PF. 2000. The causes of reduced fertility with cryopreserved semen. Anim Reprod Sci, 61:481-492.

Westendorf P, Richter L, Treu H. 1975. Deep freezing of boar spermatozoa. Laboratory and insemination results using the Hulsenberger paillete method [in German]. Dtsch Tierarztl Wochenschr, 82:261-267.

White IG, 1993. Lipids and calcium uptake of sperm in relation to cold shock and preservation. Reprod Fertil Dev, 5:639-658.

Zeng C, Peng W, Ding L, He L, Zhang Y, Fang D, Tang K. 2014. A preliminary study on epigenetic changes during boar spermatozoa cryopreservation. Cryobiology, 69:119-127.

Zhao G, Subbaiah PV, Mintzer E, Chiu SW, Jakobsson E, Scott HL. 2011. Molecular dynamic simulation study of cholesterol and conjugated double bonds in lipid bilayers. Chem Phys Lipids, 164:811-818. 